Pacific

Journal of

Mathematics

RANDOM ATTRACTOR FOR A DAMPED SINE-GORDON EQUATION WITH WHITE NOISE

XIAOMING FAN

Volume $216 \quad$ No. 1

September 2004 


\title{
RANDOM ATTRACTOR FOR A DAMPED SINE-GORDON EQUATION WITH WHITE NOISE
}

\author{
XIAOMING FAN
}

\begin{abstract}
We prove the existence of a compact random attractor for the random dynamical system generated by a damped sineGordon with white noise. And we obtain a precise estimate of the upper bound of the Hausdorff dimension of the random attractor, which decreases as the damping grows and shows that the dimension is uniformly bounded for the damping. In particular, under certain conditions, the dimension is zero.
\end{abstract}

\section{Introduction}

This article is devoted to the existence and estimate of Hausdorff dimension of the random attractor for a damped sine-Gordon equation with homomgeneous Dirichlet boundary condition when there is a random term.

Let $\Omega$ be an open bounded set of $R^{n}$ with a smooth boundary $\partial \Omega$. We consider the equation

$$
\left\{\begin{array}{l}
u_{t t}+\alpha u_{t}-\Delta u+\beta \sin u=q(x) \dot{W}, \quad \text { in } \quad \Omega \times[\tau,+\infty), \quad \tau \in R, \\
\left.u(x, t)\right|_{x \in \partial \Omega}=0, \quad t \geq \tau, \\
u(x, \tau)=u_{0}(x) \in H_{0}^{1}(\Omega), \quad u_{t}(x, \tau)=u_{1}(x) \in L^{2}(\Omega) .
\end{array}\right.
$$

where $u=u(x, t)$ is a real-valued function on $\Omega \times[\tau,+\infty)$, for $\tau \in R ; \alpha>0$ is called the damping; $q(x) \in H^{2}(\Omega) \cap H_{0}^{1}(\Omega), \dot{W}(t)$ is the derivative of a onedimensional two-sided Wiener process $W(t)$; and $q(x) \dot{W}$ formally describes white noise.

A random attractor of a random dynamical system (RDS) is a measurable and compact invariant random set attracting all the orbits. When such an attracting exists, it is the smallest attracting compact set and the largest invariant set $[3]$. This seems to be a good generalization of the now classical concept of a global attractor for deterministic dynamical systems. The notion of a random attractor is very useful for many infinite-dimensional random dynamical systems $[4,3]$.

Many authors $[8,10]$ have studied and estimated the Hausdorff dimension of the global attractor for a deterministic damped sine-Gordon equation. H. Crauel and F. Flandoli [4] introduced the notion of a random attractor and obtained a general theorem on the existence of a random attractor for an 
RDS. Their theorem has been successfully applied to the stochastic reactiondiffusion equation and the stochastic Navier-Stokes equation. H. Crauel et al. [3] generalized the notion of an attractor for the stochastic dynamical system introduced previously and considered a stochastic nonlinear wave equation. A. Debussche [6, 7] provided a general way to obtain the Hausdorff dimension of a random invariant set or random attractor and applied it to the random attractor for a stochastic reaction-diffusion equation. In this paper, we use the notion and framework in $[6,7,3]$ to study a damped sineGordon equation with white noise. We prove the existence of the random attractor of the equation and estimate its Hausdorff dimension. The upper bound of the Hausdorff dimension decreases as the damping grows, and the dimension is uniformly bounded for the damping. In particular, under certain conditions, the dimension is zero.

\section{Random dynamical systems; existence and uniqueness of solutions}

Let $(\Theta, F, P)$ be a probability space and $\left\{\theta_{t}: \Theta \rightarrow \Theta, t \in R\right\}$ a family of measure preserving transformations such that $(t, \omega) \mapsto \theta_{t} \omega$ is measurable, $\theta_{0}=\mathrm{id}$, and $\theta_{t+s}=\theta_{t} \theta_{s}$ for all $s, t \in R$. The flow $\theta_{t}$ together with the probability space $\left(\Theta, F, P,\left(\theta_{t}\right)_{t \in R}\right)$ is called as a (measurable) dynamical system.

A random dynamical system (RDS) on a Polish space $(X, d)$ with Borel $\sigma$-algebra $\mathcal{B}$ over $\theta$ on $(\Theta, F, P)$ is a measurable map

$$
\begin{aligned}
\phi: R^{+} \times \Theta \times X & \rightarrow X, \\
(t, \omega, x) & \mapsto \phi(t, \omega) x,
\end{aligned}
$$

such that $P$-almost surely $(P$-a.s. $)$ we have:

(i) $\phi(0, \omega)=$ id on $X$.

(ii) $\phi(t+s, \omega)=\phi\left(t, \theta_{s} \omega\right) \circ \phi(s, \omega)$ for all $s, t \in R^{+}$(cocycle property).

An RDS is continuous or differentiable if $\phi(t, \omega): X \rightarrow X$ is continuous or differentiable.

A map $D: \Theta \rightarrow 2^{X}$ is said to be a closed (compact) random set if $D(\omega)$ is closed (compact) for $P$-a.s. $\omega \in \Theta$ and if $\omega \mapsto d(x, D(\omega))$ is $P$-a.s. measurable for all $x \in X$.

It is well-known that the operator $A=-\Delta: D(A)=H_{0}^{1}(\Omega) \cap H^{2}(\Omega) \rightarrow$ $L^{2}(\Omega)$ is self-adjoint, positive and linear, and its eigenvalues $\left\{\lambda_{i}\right\}_{i \in N}$ (with $\lambda_{i} \leq \lambda_{j}$ for $i<j$ ) are positive and satisfy $\lambda_{m} \rightarrow+\infty$ as $m \rightarrow+\infty$. Consider $L^{2}(\Omega), H_{0}^{1}(\Omega)$ and $E=H_{0}^{1}(\Omega) \times L^{2}(\Omega)$ with the usual inner products and norms:

$$
(u, v)=\int_{\Omega} u v d x, \quad\|u\|_{0}=(u, u)^{1 / 2} \quad \text { for all } u, v \in L^{2}(\Omega),
$$




$$
\begin{gathered}
((u, v))=\int_{\Omega} \nabla u \cdot \nabla v d x, \quad\|u\|=((u, u))^{1 / 2} \quad \text { for all } u, v \in H_{0}^{1}(\Omega), \\
\left(y_{1}, y_{2}\right)_{E}=\left(\left(u_{1}, u_{2}\right)\right)+\left(v_{1}, v_{2}\right), \quad\|y\|_{E}=(y, y)_{E}^{1 / 2}, \\
\quad \text { for all } y_{i}=\left(u_{i}, v_{i}\right)^{T}, \quad y=(u, v)^{T} \in E, \quad i=1,2 .
\end{gathered}
$$

It is convenient to reduce (1) to an evolution equation of the first-order in time:

$$
\left\{\begin{array}{l}
u_{t}=v \\
v_{t}=-\alpha v+\Delta u-\beta \sin u+q(x) \dot{W}, \\
u(x, \tau)=u_{0}(x), \quad v(x, \tau)=u_{1}(x), \quad x \in \Omega,
\end{array}\right.
$$

whose equivalent Itó equation is

$$
\left\{\begin{array}{l}
d u=v d t \\
d v=-\alpha v d t+\Delta u d t-\beta \sin u d t+q(x) d W \\
u(x, \tau)=u_{0}(x), \quad v(x, \tau)=u_{1}(x), \quad x \in \Omega
\end{array}\right.
$$

$W(t)$ is a one-dimensional two-sided Wiener process with path $\omega(\cdot)$ in the space $C(R, R)$ of continuous functions on $R, \omega(0)=0$. We can define a family of measure-preserving and ergodic transformations (a flow) $\left\{\theta_{t}\right\}_{t \in R}$ by

$$
\theta_{t} \omega(\cdot)=\omega(\cdot+t)-\omega(t) .
$$

Let $z=v-q(x) W$, then $v=z+q(x) W$. We consider the random partial differential equation equivalent to (3)

$$
\left\{\begin{array}{l}
d u / d t=z+q(x) W \\
d z / d t=-\alpha z+\Delta u-\beta \sin u-\alpha q(x) W \\
u(x, \tau)=u_{0}(x), \quad z(\tau, \omega)=z(x, \tau, \omega)=u_{1}(x)-q(x) W(\tau), \quad x \in \Omega
\end{array}\right.
$$

In contrast to the stochastic differential equation (2), no stochastic differential appears here. Let

$$
\varphi=\left(\begin{array}{l}
u \\
z
\end{array}\right), \quad L=\left(\begin{array}{cc}
0 & I \\
-A & -\alpha I
\end{array}\right), \quad F(\varphi, \omega)=\left(\begin{array}{c}
q(x) W \\
-\beta \sin u-\alpha q(x) W
\end{array}\right) .
$$

Then (4) can be written as

$$
\dot{\varphi}=L \varphi+F(\varphi, \omega), \quad \varphi(\tau, \omega)=\left(u_{0}, z(\tau, \omega)\right)^{T} .
$$

We know that $L$ is the infinitesimal generator of a $C_{0}$-semigroup $e^{L t}$ on $E$ with the exponential dichotomy from [9]. It is easy to check that the function $F(\cdot, \omega): E \mapsto E$ is globally Lipschitz continuous with respect to $\varphi$ and bounded for every $\omega \in \Theta$. By the classical semigroup theory of existence and uniqueness of the solutions of evolution differential equations [9], the unique solution of (5) can be interpreted in a mild sense:

$$
\varphi(t, \omega)=e^{L(t-\tau)} \varphi(\tau, \omega)+\int_{\tau}^{t} e^{L(t-s)} F(\varphi(s), \omega) d s,
$$


surely for any $\varphi(\tau, \omega) \in E$. One can show that for $P$-a.s. every $\omega \in \Theta$ the following statements hold for all $T>0$ :

(i) If $\varphi(\tau, \omega) \in E$ then $\varphi(t, \omega)$ lies in

$$
C\left([\tau, \tau+T) ; H_{0}^{1}(\Omega)\right) \times C\left([\tau, \tau+T) ; L^{2}(\Omega)\right) .
$$

(ii) $\varphi(t, \varphi(\tau, \omega))$ is jointly continuous in $t$ and $\varphi(\tau, \omega)$.

(iii) The solution mapping of (5) satisfies the properties of an RDS.

This equation has a unique solution for every $\omega \in \Theta$. No exceptional sets appear. Hence the solution mapping

$$
\hat{S}(t, \omega): \varphi(\tau, \omega) \mapsto \varphi(t, \omega)
$$

generates a random dynamical system. So the transformation

$$
S(t, \omega): \varphi(\tau, \omega)+(0, q(x) W(\tau))^{T} \mapsto \varphi(t, \omega)+(0, q(x) W(t))^{T}
$$

also determines a random dynamical system corresponding to problem (2).

We will prove the existence of a nonempty compact random attractor for the random dynamical system $S(t, \omega)$ and estimate the Hausdorff dimension of the random attractor.

\section{Existence of a random attractor}

A random set $K(\omega)$ is said to absorb the set $B \subset X$ for an $\operatorname{RDS} \varphi$ if $P$-a.s. there exists $t_{B}(\omega)$ such that

$$
\varphi\left(t, \theta_{-t} \omega\right) B \subset K(\omega) \text { for all } t \geq t_{B}(\omega) .
$$

A random set $A(\omega)$ is said to be a random attractor associated to the RDS $\varphi$ if $P$-a.s.:

(i) $A(\omega)$ is a random compact set, that is, $P$-a.s. $\omega \in \Theta, A(\omega)$ is compact and for all $x \in X$ and $P$-a.s. the map $x \mapsto \operatorname{dis}(x, A(\omega))$ is measurable.

(ii) $\varphi(t, \omega) A(\omega)=A\left(\theta_{t} \omega\right)$ for all $t \geq 0$ (invariance).

(iii) For all bounded (and nonrandom) $B \subset X$,

$$
\lim _{t \rightarrow \infty} \operatorname{dis}\left(\varphi\left(t, \theta_{-t} \omega\right) B, A(\omega)\right)=0
$$

where $\operatorname{dis}(\cdot, \cdot)$ denotes the Hausdorff semidistance:

$$
\operatorname{dis}(A, B)=\sup _{x \in A} \inf _{y \in B} d(x, y), \quad A, B \in X .
$$

Note that $\varphi\left(t, \theta_{-t} \omega\right) x$ can be interpreted as the position at $t=0$ of the trajectory which was in $x$ at time $-t$. Thus, the attraction property holds from $t=-\infty$. 
Theorem 1 (Existence of a random attractor). Let $\phi$ be an RDS on a Polish space $(X, d)$ with Borel $\sigma$-algebra $\mathcal{B}$ over the flow $\left\{\theta_{t}\right\}_{t \in R}$ on a probability space $(\Theta, F, P)$. Suppose there exists a random compact set $K(\omega)$ such that for any bounded nonrandom set $B \subset X P$-a.s.

$$
\operatorname{dis}\left(\varphi\left(t, \theta_{-t} \omega\right) B, K(\omega)\right) \rightarrow 0 \quad \text { as } t \rightarrow+\infty .
$$

Then the set

$$
A(\omega)=\overline{\bigcup_{B \subset X} \Lambda(\omega)}
$$

is a random attractor for $\phi$, where the union is taken over all bounded $B \subset$ $X$, and $\Lambda_{B}(\omega)$ is the omega-limit set of $B$ given by

$$
\Lambda_{B}(\omega)=\bigcap_{s \geq 0} \overline{\bigcup_{t \geq s} \phi\left(t, \theta_{-t} \omega\right) B} .
$$

Moreover, the random attractor is unique.

Proof. Since $K(\omega)$ is a random compact set, then by (8), $\Lambda_{B}(\omega)$ is also random compact and nonempty. By the proof of Theorem 3.11 in $[4], A(\omega)$ is a random attractor for $\phi$ and it is unique.

Remark 1. Theorem 1 can be regarded as an analog of [2, Theorem 2.2]. As in [2], the RDS $\phi$ can be also said to be uniformly asymptotically compact.

We show the existence of a random attractor for the RDS (7) in the space $E$. Let $\psi=(u, \bar{z})^{T}, \bar{z}=z+\varepsilon u$, where

$$
\varepsilon=\frac{\alpha \lambda_{1}}{2 \alpha^{2}+3 \lambda_{1}}
$$

Then the system (4) can be written as

$$
\psi_{t}+Q \psi=\bar{F}(\psi, \omega), \quad \psi(\tau, \omega)=\left(u_{0}, z(\tau, \omega)+\varepsilon u_{0}\right)^{T}, \quad t \geq \tau,
$$

where

$$
\begin{aligned}
Q & =\left(\begin{array}{cc}
\varepsilon I & -I \\
A-\varepsilon(\alpha-\varepsilon) I & (\alpha-\varepsilon) I
\end{array}\right), \\
\bar{F}(\psi, \omega) & =\left(\begin{array}{c}
q(x) W \\
-\beta \sin u-(\alpha-\varepsilon) q(x) W
\end{array}\right) .
\end{aligned}
$$

The mapping

$$
\hat{S}_{\varepsilon}(t, \omega):\left(u_{0}, z(\tau, \omega)+\varepsilon u_{0}\right)^{T} \mapsto(u(t), z(t)+\varepsilon u(t))^{T}, \quad E \mapsto E, \quad t \geq \tau
$$

defined by (10) has the following relation with $\hat{S}(t, \omega)$ :

$$
\hat{S}_{\varepsilon}(t, \omega)=R_{\varepsilon} \hat{S}(t, \omega) R_{-\varepsilon}
$$


where $R_{\varepsilon}:(u, z)^{T} \mapsto(u, z+\varepsilon u)^{T}$ is an isomorphism of $E$. So, for the RDS (7) we only need consider the equivalent random dynamical system $S_{\varepsilon}(t, \omega)=R_{\varepsilon} S(t, \omega) R_{-\varepsilon}$, where $S_{\varepsilon}(t, \omega)$ is decided by

$$
\vartheta_{t}+Q \vartheta=G(\vartheta, \omega), \quad \vartheta(\tau)=\left(u_{0}, u_{1}+\varepsilon u_{0}\right)^{T}, \quad t \geq \tau,
$$

where $\vartheta(t)=\left(u(t), u_{t}(t)+\varepsilon u(t)\right)^{T}$ and

$$
G(\vartheta, \omega)=\left(\begin{array}{c}
0 \\
-\beta \sin u+q(x) \dot{W}
\end{array}\right) .
$$

First we present a positivity property of the operator $Q$ in $E$ that plays an important role in this article.

Lemma 1. For any $\varphi=(u, v)^{T} \in E$,

$$
(Q \varphi, \varphi)_{E} \geq \frac{\varepsilon}{2}\|\varphi\|_{E}^{2}+\frac{\varepsilon}{4}\|u\|^{2}+\frac{\alpha}{2}\|v\|_{0}^{2} .
$$

Proof. This is easily obtained after simple computations.

Lemma 2. There exist a random variable $r_{1}(\omega)>0$ and a bounded ball $B_{0}$ of $E$ centered at 0 with random radius $r_{0}(\omega)$ such that for any bounded nonrandom set $B$ of $E$, there exists a deterministic $T(B) \leq-1$ such that the solution $\psi(t, \omega ; \psi(\tau, \omega))=(u(t, \omega), \bar{z}(t, w))^{T}$ of $(10)$ with initial value $\left(u_{0}, u_{1}+\varepsilon u_{0}\right)^{T} \in B$ satisfies for P-a.s. $\omega \in \Theta$

$$
\|\psi(-1, \omega ; \psi(\tau, \omega))\|_{E} \leq r_{0}(\omega), \quad \tau \leq T(B),
$$

and for $\tau \leq t \leq 0$,

$$
\begin{aligned}
\| \psi(t, \omega ; & \psi(\tau, \omega)) \|_{E}^{2} \\
& \leq 2\left(e^{-\varepsilon(t-\tau)}\left(\left\|u_{0}\right\|^{2}+\left\|u_{1}+\varepsilon u_{0}\right\|_{0}^{2}+\|q\|_{0}^{2}|W(\tau)|^{2}\right)+r_{1}^{2}(\omega)\right),
\end{aligned}
$$

where $\bar{z}(t, w)=u_{t}(t)+\varepsilon u(t)-q(x) W(t)$.

Of course one can deduce a similar absorption result for

$$
\vartheta(-1)=\left(u(-1), u_{t}(-1)+\varepsilon u(-1)\right)^{T}
$$

instead of $\psi(-1)$.

Proof. Taking the inner product $(\cdot, \cdot)_{E}$ of $(10)$ with $\psi=(u, v)^{T}$, in which $v=u_{t}+\varepsilon u-q W$, we obtain

$$
\begin{aligned}
& \frac{1}{2} \frac{d}{d t}\|\psi\|_{E}^{2}+(Q \psi, \psi)_{E} \\
& \quad=(-\beta \sin u, v)-(\alpha-\varepsilon)(q(x), v) W(t)+((q(x), u)) W(t), \quad t \geq \tau .
\end{aligned}
$$

By Young's inequality and Lemma 1

$$
\frac{d}{d t}\|\psi\|_{E}^{2}+\varepsilon\|\psi\|_{E}^{2} \leq 2\left(\frac{\beta^{2}}{\alpha}+\alpha\|q\|_{0}^{2}|W(t)|^{2}+\frac{\|q\|^{2}}{\varepsilon}|W(t)|^{2}\right), \quad t \geq \tau .
$$


By the Gronwall lemma

$$
\begin{aligned}
\| \psi(t, \omega & ; \psi(\tau, \omega)) \|_{E}^{2} \\
\leq & \left.e^{-\varepsilon(t-\tau)} \| \psi(\tau, \omega)\right) \|_{E} \\
& +2 \int_{\tau}^{t} e^{-\varepsilon(t-s)}\left(\frac{\beta^{2}}{\alpha}+\alpha\|q\|_{0}^{2}|W(s)|^{2}+\frac{\|q\|^{2}}{\varepsilon}|W(s)|^{2}\right) d s \\
\leq & 2 e^{-\varepsilon(t-\tau)}\left(\left\|u_{0}\right\|^{2}+\left\|u_{1}+\varepsilon u_{0}\right\|_{0}^{2}+\|q\|_{0}^{2}|W(\tau)|^{2}\right) \\
& +2 \int_{\tau}^{t} e^{-\varepsilon(t-s)}\left(\frac{\beta^{2}}{\alpha}+\left(\alpha\|q\|_{0}^{2}+\frac{\|q\|^{2}}{\varepsilon}\right)|W(s)|^{2}\right) d s .
\end{aligned}
$$

Put

$$
\begin{aligned}
& r_{0}^{2}(\omega)=2\left(1+\frac{\beta^{2}}{\varepsilon \alpha}+\sup _{\tau \leq-1} e^{\varepsilon s}\|q\|_{0}^{2}|W(\tau)|^{2}\right. \\
& \left.\quad+\left(\alpha\|q\|_{0}^{2}+\frac{\|q\|^{2}}{\varepsilon}\right) \int_{-\infty}^{-1} e^{-\varepsilon(-1-s)}|W(s)|^{2} d s\right)
\end{aligned}
$$

and

$$
r_{1}^{2}(\omega)=\frac{\beta^{2}}{\varepsilon \alpha}+\left(\alpha\|q\|_{0}^{2}+\frac{\|q\|^{2}}{\varepsilon}\right) \int_{-\infty}^{0} e^{\varepsilon s}|W(s)|^{2} d s .
$$

Since $\lim _{t \rightarrow \infty} W(t) / t=0$, the quantities $r_{0}^{2}(\omega)$ and $r_{1}^{2}(\omega)$ are finite $P$-a.s. Given a bounded set $B$ of $E$, choose $T(B) \leq-1$ such that

$$
e^{-\varepsilon(-1-\tau)}\left(\left\|u_{0}\right\|^{2}+\left\|u_{1}+\varepsilon u_{0}\right\|_{0}^{2}\right) \leq 1 \quad \text { for all }\left(u_{0}, u_{1}+\varepsilon u_{0}\right)^{T} \in B
$$

and

$$
-\tau e^{\varepsilon \tau}\left(\left\|u_{0}\right\|^{2}+\left\|u_{1}+\varepsilon u_{0}\right\|_{0}^{2}\right) \leq 1 \quad \text { for all }\left(u_{0}, u_{1}+\varepsilon u_{0}\right)^{T} \in B
$$

for all $\tau \leq T(B)$. The proof is completed from (16).

Let $u(t)$ be a solution of system (1) with initial value $\left(u_{0}, u_{1}+\varepsilon u_{0}\right)^{T} \in B$. We make the decomposition $u(t)=y_{1}(t)+y_{2}(t)$, where $y_{1}$ and $y_{2}$ satisfy

$$
\left\{\begin{array}{l}
y_{1 t t}+\alpha y_{1 t}-\Delta y_{1}=0 \quad \text { in } \Omega \times[\tau,+\infty) \\
\left.y_{1}(x, t)\right|_{x \in \partial \Omega}=0, \quad t \geq \tau, \\
y_{1}(x, \tau)=u_{0}(x), \quad y_{1 t}(x, \tau)=u_{1}(x), \quad x \in \Omega
\end{array}\right.
$$

and

$$
\left\{\begin{array}{l}
y_{2 t t}+\alpha y_{2 t}-\Delta y_{2}+\beta \sin u=q(x) \dot{W} \quad \text { in } \Omega \times[\tau,+\infty) \\
\left.y_{2}(x, t)\right|_{x \in \partial \Omega}=0, \quad t \geq \tau, \\
y_{2}(x, \tau)=y_{2 t}(x, \tau)=0, \quad x \in \Omega
\end{array}\right.
$$

Lemma 3. Let $B$ be a bounded nonrandom subset of $E$. We have, for any $\left(u_{0}, u_{1}+\varepsilon u_{0}\right)^{T} \in B$,

$$
\left\|Y_{1}(0)\right\|_{E}^{2}=\left\|y_{1}(0)\right\|^{2}+\left\|y_{1 t}(0)+\varepsilon y_{1}(0)\right\|_{0}^{2} \leq\left(\left\|u_{0}\right\|^{2}+\left\|u_{0}+\varepsilon u_{1}\right\|_{0}^{2}\right) e^{\varepsilon \tau},
$$


and there exists a random radius $r_{2}(\omega)$ such that for P-a.s. $\omega \in \Theta$,

$$
\left\|A^{1 / 2} Y_{2}\left(0, \omega ; Y_{2}(\tau, \omega)\right)\right\|_{E}^{2} \leq r_{2}^{2}(\omega),
$$

where $Y_{1}=\left(y_{1}, y_{1 t}+\varepsilon y_{1}\right)^{T}$ and $Y_{2}=\left(y_{2}, y_{2 t}+\varepsilon y_{2}-q(x) W\right)$ satisfy (18), (19).

Proof. Take the inner product of $(18)$ in $L^{2}(\Omega)$ with $y_{1 t}+\varepsilon y_{1}$ whose initial value is $\left(u_{0}, u_{1}+\varepsilon u_{0}\right)^{T}$. After a simple computation using Lemma 1 , one obtains (20).

Set $Y_{2}=\left(y_{2}, y_{2 t}+\varepsilon y_{2}-q(x) W\right)^{T}$. Equation (19) can be reduced to

$$
Y_{2 t}+Q Y_{2}=H\left(Y_{2}, \omega\right), \quad Y_{2}(\tau)=(0,-q(x) W(\tau)), \quad t \geq \tau
$$

where

$$
H\left(Y_{2}, \omega\right)=\left(\begin{array}{c}
q(x) W(t) \\
-\beta \sin u-(\alpha-\varepsilon) q(x) W(t)
\end{array}\right) .
$$

Taking the inner product of (22) in $E$ with $A Y_{2}$ and using Young's inequality, Lemma 1 and (14), we obtain for $\tau \leq T(B)$

$$
\begin{aligned}
\frac{d}{d t}\left\|A^{1 / 2} Y_{2}\right\|_{E}^{2}+\varepsilon\left\|A^{1 / 2} Y_{2}\right\|_{E}^{2} & \\
\leq & \frac{4 \beta^{2}}{\alpha}\left(e^{-\varepsilon(t-\tau)}\left(\left\|u_{0}\right\|^{2}+\left\|u_{1}+\varepsilon u_{0}\right\|_{0}^{2}+\|q\|_{0}^{2}|W(\tau)|^{2}\right)+r_{1}^{2}(\omega)\right) \\
& +2\left(\alpha\|q\|^{2}+\frac{\|A q\|_{0}^{2}}{\varepsilon}\right)|W(t)|^{2}, \quad \tau \leq t \leq 0 .
\end{aligned}
$$

By the Gronwall lemma,

$$
\begin{aligned}
\| A^{1 / 2} & Y_{2}\left(0, \omega ; Y_{2}(\tau, \omega)\right) \|_{E}^{2} \\
\leq & \frac{4 \beta^{2}}{\varepsilon \alpha}\left((-\tau) e^{\varepsilon \tau}\left(\left\|u_{0}\right\|^{2}+\left\|u_{1}+\varepsilon u_{0}\right\|_{0}^{2}+\|q\|_{0}^{2}|W(\tau)|^{2}\right)+r_{1}^{2}(\omega)\right) \\
& \quad+e^{\varepsilon \tau}\|q\|^{2}|W(\tau)|^{2}+2\left(\alpha\|q\|^{2}+\frac{\|A q\|_{0}^{2}}{\varepsilon}\right) \int_{\tau}^{0} e^{\varepsilon s}|W(s)|^{2} d s .
\end{aligned}
$$

Put

$$
\begin{aligned}
r_{2}^{2}(\omega)= & \frac{4 \beta^{2}}{\varepsilon \alpha}\left(1+\|q\|_{0}^{2} \sup _{\tau \leq 0}(-\tau) e^{\varepsilon \tau}|W(\tau)|^{2}+r_{1}^{2}(\omega)\right) \\
& +\|q\|^{2} \sup _{\tau \leq 0} e^{\varepsilon \tau}|W(\tau)|^{2}+2\left(\alpha\|q\|^{2}+\frac{\|A q\|_{0}^{2}}{\varepsilon}\right) \int_{-\infty}^{0} e^{\varepsilon s}|W(s)|^{2} d s .
\end{aligned}
$$

Since $\lim _{t \rightarrow \infty} W(t) / t=0$, the quantity $r_{2}^{2}(\omega)$ is finite $P$-a.s. By $(17)$ and (23), we have

$\left\|A^{1 / 2} Y_{2}\left(0, \omega ; Y_{2}(\tau, \omega)\right)\right\|_{E}^{2} \leq r_{2}^{2}(\omega) \quad$ for all $\left(u_{0}, u_{1}+\varepsilon u_{0}\right)^{T} \in B, \quad \tau \leq T(B)$.

The proof is complete. 
Let $B_{1 / 2}(w)$ be the ball of $D(A) \times H_{0}^{1}(\Omega)$ of radius $r_{1}(\omega)$. From the compact embedding $D(A) \times H_{0}^{1}(\Omega) \hookrightarrow E$ we see that $B_{1 / 2}(w)$ is compact. For every bounded nonrandom set $B$ of $E$, Pick any $\psi(0) \in \hat{S}_{\varepsilon}\left(t, \theta_{-t} \omega\right) B$. From Lemma 3, we have $Y_{2}(0)=\psi(0)-Y_{1}(0) \in B_{1 / 2}(w)$ where $Y_{2}(t, \omega)$ is given by (22). Therefore, again by Lemma 3 ,

$\inf _{\ell(0) \in B_{1 / 2}(w)}\|\psi(0)-\ell(0)\|_{E}^{2} \leq\left\|Y_{1}(0)\right\|_{E}^{2} \leq\left(\left\|u_{0}\right\|^{2}+\left\|u_{0}+\varepsilon u_{1}\right\|_{0}^{2}\right) e^{\varepsilon \tau}, \quad \tau \leq 0$.

So $\operatorname{dis}\left(\hat{S}_{\varepsilon}\left(t, \theta_{-t} \omega\right) B, B_{1 / 2}(w)\right) \leq\left(\left\|u_{0}\right\|^{2}+\left\|u_{0}+\varepsilon u_{1}\right\|_{0}^{2}\right) e^{-\varepsilon t}$, for all $t \geq 0$.

From the relation (12) between $S_{\varepsilon}(t, \omega)$ and $\hat{S}_{\varepsilon}(t, \omega)$, one can easily obtain that for any nonrandom bounded $B \subset E P$-a.s.

$$
\operatorname{dis}\left(S_{\varepsilon}\left(t, \theta_{-t} \omega\right) B, B_{1 / 2}(w)\right) \rightarrow 0 \quad \text { as } t \rightarrow+\infty .
$$

Corollary 1. The RDS $S_{\varepsilon}(t, \omega)$ associated with (7) possesses a uniformly attracting compact set $B_{1 / 2}(w) \subset E$. So the $\operatorname{RDS} S_{\varepsilon}(t, \omega)$ is uniformly asymptotically compact in $E$.

Theorem 2. The RDS $S_{\varepsilon}(t, \omega)$ has a nonempty compact random attractor $A(\omega)$.

Proof. This follows from Lemmas 2 and 3 and Corollary 1.

\section{Hausdorff dimension of the random attractor}

To bound the attractor's dimension we use the following result of Debussche $[6,7]$. He treats the case of a random attractor $A(\omega)$ invariant under a random map $S(t, \omega)$ : for some measure-preserving ergodic transformation $\theta$ on $(\Theta, F, \mathcal{P})$ we have

$$
S(\omega) A(\omega)=A(\theta \omega) .
$$

One must make some assumptions about the map $S(t, \omega)$ : first, we need $S(\omega)$ to be almost surely uniformly differentiable on $A(\omega)$, which means that $P$-almost surely, for every $u \in A(\omega)$, there exists a bounded linear operator $D S(u, \omega): X \rightarrow X$ such that $u+h \in A(\omega)$ implies

$$
|S(\omega)(u+h)-S(\omega) u-D S(u, \omega) h| \leq K(\omega)|h|^{1+\delta},
$$

where $\delta>0$ and $K(\omega)$ is a random variable with $K(\omega) \geq 1$ and $E(\log K)<$ $\infty$. Given a bounded linear operator $L$ on $X$ and $n \in N$, we set

$$
\alpha_{n}(L)=\sup _{\substack{G \subset X \\ \operatorname{dim} G \leq n}} \inf _{\phi \in G}|L \phi|
$$

and

$$
\epsilon_{n}(L)=\alpha_{1}(L) \cdots \alpha_{n}(L)
$$

Assume that

$$
\epsilon_{d}(D S(u, \omega)) \leq \bar{\epsilon}(\omega)
$$


where $\bar{\epsilon}(\omega)$ is a random variable satisfying $E(\log \bar{\epsilon}(\omega))<0$ and the additional (relatively easy) condition that, for some random variable $\bar{\alpha}(\omega) \geq 1$, we have

$$
\alpha_{1}(D S(u, \omega)) \leq \bar{\alpha}(\omega) \quad \text { with } E(\log \bar{\alpha}(\omega))<\infty .
$$

Under these assumptions, the Hausdorff dimension satisfies $d_{H}(A(\omega))<d$ almost surely.

Lemma 4. Consider the linearized equation of (13) with initial boundary conditions

$$
\Phi_{t}+Q \Phi=G_{\vartheta}^{\prime}(\vartheta, \omega) \Phi, \quad \Phi(0)=(\xi, \eta)^{T}, \quad t \geq 0,
$$

where $\Phi=(U, V)^{T} \in E$ and $\vartheta(t)=\left(u(t), u_{t}(t)+\varepsilon u(t)\right)^{T} \in E, t \geq 0$ is the solution of (13) with initial value $\vartheta(0)=\left(u_{0}, u_{1}+\varepsilon u_{0}\right)^{T}$, and

$$
G_{\vartheta}^{\prime}(\vartheta, \omega)=\left(\begin{array}{cc}
0 & 0 \\
-\beta \cos u & 0
\end{array}\right) \text {. }
$$

Then (25) is a P-a.s. well-posed problem in $E$ and $S_{\varepsilon}(t, \omega)$ is uniformly differentiable for $P$-a.s. $\omega \in \Theta$ on the random attractor $A(\omega)$, with differential $D S_{\varepsilon}(\vartheta(0), t, \omega)(\xi, \eta)^{T}=\Phi(t, \omega): E \mapsto E$ a bounded linear operator satisfying $P$-a.s.

(26) $\left\|S_{\varepsilon}(t, \omega)\left(\vartheta(0)+(\xi, \eta)^{T}\right)-S_{\varepsilon}(t, \omega) \vartheta(0)-D S_{\varepsilon}(\vartheta(0), t, \omega)(\xi, \eta)^{T}\right\|_{E}$

$$
\leq k(t)\left\|(\xi, \eta)^{T}\right\|_{E}^{2},
$$

where $K(t) \geq 1$ is independent of $\omega, t \geq 0$.

Proof. It is clear that the problem (25) is $P$-a.s. well-posed in $E$.

We consider the Lipschitz property of $S_{\varepsilon}(t, \omega)$. Set

$$
\begin{aligned}
& \vartheta(t)=S_{\varepsilon}(t, \omega) \vartheta_{0}=\left(u(t), u_{t}(t)+\varepsilon u(t)\right)^{T}, \\
& \bar{\vartheta}(t)=S_{\varepsilon}(t, \omega)\left(\vartheta(0)+(\xi, \eta)^{T}\right)=\left(\bar{u}(t), \bar{u}_{t}(t)+\varepsilon \bar{u}(t)\right)^{T} .
\end{aligned}
$$

Let $\widetilde{\vartheta}(t)=\bar{\vartheta}(t)-\vartheta(t)$, which satisfies

$$
\tilde{\vartheta}_{t}+Q \widetilde{\vartheta}=\left(\begin{array}{c}
0 \\
-\beta(\sin \bar{u}-\sin u)
\end{array}\right), \quad \widetilde{\vartheta}(0)=(\xi, \eta)^{T}, \quad t \geq 0 .
$$

Taking the inner product of (27) with $\widetilde{\vartheta}$ in $E$ we have, after a simple computation,

$$
\|\widetilde{\vartheta}(t)\|_{E}=\|\bar{\vartheta}(t)-\vartheta(t)\|_{E} \leq\left\|(\xi, \eta)^{T}\right\|_{E} e^{|\beta| t} .
$$

Next we show the differentiability of the $\operatorname{RDS} S_{\varepsilon}(t, \omega)$. Let $Z(t)=\bar{\vartheta}(t)-$ $\vartheta(t)-\Phi(t)$. Then $Z(t)$ satisfies

$$
Z_{t}+Q Z=\left(\begin{array}{c}
0 \\
-\beta(\sin \bar{u}-\sin u-\cos u U)
\end{array}\right), \quad Z(0)=0, \quad t \geq 0 .
$$


It is easily checked that there exists a deterministic constant $\delta>0$ such that

$$
|\sin \bar{u}-\sin u-\cos u U| \leq|\bar{u}-u|^{2}+|\bar{u}-u-U| .
$$

Taking the inner product of (29) with $Z$ in $E$, by the preceging inequality and Young's inequality we see that there exists a deterministic constant $\gamma_{1}>0$ such that

$$
\frac{d}{d t}\|Z(t)\|_{E}^{2} \leq \gamma_{1}\|Z(t)\|_{E}^{2}+\gamma_{1}\|\widetilde{\vartheta}(t)\|_{E}^{4}
$$

From (28),

$$
\frac{d}{d t}\|Z(t)\|_{E}^{2} \leq \gamma_{1}\|Z(t)\|_{E}^{2}+\gamma_{1}\left\|(\xi, \eta)^{T}\right\|_{E}^{4} e^{4|\beta| t} .
$$

By the Gronwall lemma and zero initial value at $t=\tau$, there exist deterministic constants $\gamma_{2}, \gamma_{3}>0$ such that

$$
\|\bar{\vartheta}(t)-\vartheta(t)-\Phi(t)\|_{E}^{2} \leq \gamma_{2} e^{\gamma_{3} t}\left\|(\xi, \eta)^{T}\right\|_{E}^{4}
$$

The proof is complete.

Lemma 5. Let $\left\{\left(\xi_{j}, \omega_{j}\right)^{T}\right\}_{j=1}^{m}$ be an orthonormal family of elements of $\left(E,\|\cdot\|_{E}\right)$. We have

$$
\sum_{j=1}^{m}\left\|\xi_{j}\right\|_{0}^{2} \leq \sum_{j=1}^{m} \lambda_{j}^{-1}
$$

Proof. This is a direct consequence of [10, Lemma VI.6.3].

Theorem 3. If

$$
\beta^{2}>\frac{\alpha^{2} \lambda_{1}}{2\left(2 \alpha^{2}+3 \lambda_{1}\right)},
$$

the Hausdorff dimension of the random attractor $A(\omega)$ for the RDS (7) satisfies

$$
d_{H}(A(\omega)) \leq \min \left\{m \in N \mid \frac{1}{m} \sum_{j=1}^{m} \lambda_{j}^{-1}<\frac{2 \alpha^{2} \lambda_{1}}{2 \beta^{2}\left(2 \alpha^{2}+3 \lambda_{1}\right)-\alpha^{2} \lambda_{1}}\right\} .
$$

Otherwise $d_{H}(A(\omega))=0$.

Proof. We apply Debussche's result. Let $D S_{\varepsilon}(\vartheta(0), \omega)=D S_{\varepsilon}(\vartheta(0), 1, \omega)$. Firstly, check that there exists a deterministic constant $\bar{\alpha} \geq 1$ such that $\alpha_{1}\left(D S_{\varepsilon}(\vartheta(0), \omega)\right) \leq \bar{\alpha}$ from $(26)$.

To find a $d$ such that $\epsilon_{d}\left(D S_{\varepsilon}(\vartheta(0), \omega)\right)<1$, we use the trace formula (see also Témam [10], Chapter V). This allows us to write $\epsilon_{d}$ in another way more dependent on the dynamics. Since $D S_{\varepsilon}(\vartheta(0), \omega)(\xi, \eta)^{T}$ is the solution of the linear equation

$$
d \Phi / d t=M(t, \vartheta(t)) \Phi, \quad \Phi(0)=(\xi, \eta)^{T},
$$


where

$$
M(t, \vartheta(t))=-Q+G_{\vartheta}^{\prime}(\vartheta, \omega)
$$

and $\vartheta(t)$ is the solution of $(13)$ with $\vartheta(0)=\left(u_{0}, u_{1}+\varepsilon u_{0}\right)^{T}$, we can write

$$
D S_{\varepsilon}(\vartheta(0), \omega)=\exp \left(\int_{0}^{1} M(s, \vartheta(s)) d s\right) .
$$

Let $\left\{\Phi_{j}\right\}_{j=1}^{m}$ be $m$ solutions of (25) with initial values $\Phi_{j}(0), j=1,2, \ldots, m$. Let $P(s)$ be an orthogonal projector of rank $m$ at the time $t=s$, onto the space spanned by $\left\{\Phi_{j}\right\}_{j=1}^{m}$ in $E$. Then

$$
\epsilon_{d}\left(D S_{\varepsilon}(\vartheta(0), \omega)\right)=\sup _{P(0)} \exp \left(\operatorname{Tr} \int_{0}^{1} M(s, \vartheta(s)) P(s) d s\right) .
$$

Let $\left.\left\{\Psi_{j}=\left(\xi_{j}, \eta_{j}\right)^{T}\right)\right\}_{j=1}^{m}$ be a standard orthonormal basis of the space spanned by $\left\{\Phi_{j}\right\}_{j=1}^{m}$. By Young's inequality and Lemma 1, We have

$$
\begin{aligned}
\left(M(s, \vartheta(s)) \Psi_{j}, \Psi_{j}\right)_{E} & =-\left(Q \Psi_{j}, \Psi_{j}\right)_{E}-\left(G_{\vartheta}^{\prime}(\vartheta, \omega) \Psi_{j}, \Psi_{j}\right)_{E} \\
& \leq-\frac{\varepsilon}{2}+\left(\frac{\beta^{2}}{2 \alpha}-\frac{\varepsilon}{4}\right)\left\|\xi_{j}\right\|_{0}^{2} .
\end{aligned}
$$

If $\beta^{2}>\frac{\alpha^{2} \lambda_{1}}{2\left(2 \alpha^{2}+3 \lambda_{1}\right)}$, by (33) and (30) of Lemma 5 ,

$$
\begin{aligned}
\operatorname{Tr}(M(s, \vartheta(s)) P(s)) & =\sum_{j=1}^{m}\left(M(s, \varphi(s)) \Phi_{j}, \Phi_{j}\right)_{E} \\
& \leq-\frac{\varepsilon m}{2}+\left(\frac{\beta^{2}}{2 \alpha}-\frac{\varepsilon}{4}\right) \sum_{j=1}^{m} \lambda_{j}^{-1} .
\end{aligned}
$$

If there exists a number $m \in N$ such that

$$
\frac{1}{m} \sum_{j=1}^{m} \lambda_{j}^{-1}<\frac{2 \alpha^{2} \lambda_{1}}{2 \beta^{2}\left(2 \alpha^{2}+3 \lambda_{1}\right)-\alpha^{2} \lambda_{1}},
$$

by (32) and (34), we obtain $E\left(\log \epsilon_{d}\left(D S_{\varepsilon}(\vartheta(0), \omega)\right)<0\right.$, then $d_{H}(A(\omega)) \leq$ $m$. If $\beta^{2} \leq \frac{\alpha^{2} \lambda_{1}}{2\left(2 \alpha^{2}+3 \lambda_{1}\right)}$, then we deduce from (33) that

$$
\left(M(s, \vartheta(s)) \Psi_{j}, \Psi_{j}\right)_{E}<0, \quad j=1,2, \ldots, m .
$$

Then $d_{H}(A(\omega))=0$.

Corollary 2. If

$$
\frac{\alpha^{2} \lambda_{1}}{2\left(2 \alpha^{2}+3 \lambda_{1}\right)}<\beta^{2}<\frac{\alpha^{2} \lambda_{1}\left(2 \lambda_{1}+1\right)}{2\left(2 \alpha^{2}+3 \lambda_{1}\right)},
$$

the Hausdorff dimension of the random attractor $A(\omega)$ for the RDS (7) satisfies $d_{H}(A(\omega))=0$. 
Remark 2. The upper bound in the right side of (31) decreases as $\alpha$ grows because the function $1 / m \sum_{j=1}^{m} \lambda_{j}^{-1}$ is decreasing in $m$ and tends to zero as $m \rightarrow \infty$, while the function

$$
\frac{2 \alpha^{2} \lambda_{1}}{2 \beta^{2}\left(2 \alpha^{2}+3 \lambda_{1}\right)-\alpha^{2} \lambda_{1}}
$$

is increasing and uniformly bounded in $\alpha$. So, the dimension $d_{H}(A(\omega))$ is uniformly bounded for the damping $\alpha$.

Acknowledgements. The author thanks Prof. Daoyi Xu and Prof. Shengfan Zhou for their advice.

\section{References}

[1] T. Caraballo and J.A. Langa, Stability and random attractors for a reaction-diffusion equation with multiplicative noise, Discrete Contin. Dynam. Systems, 6 (2000), 875892, MR 1788258 (2001j:37093), Zbl 1011.37031.

[2] V. Chepyzhov and M. Vishik, A Hausdorff dimension estimate for kernel sections of nonautonomous evolution equations, Indiana Univ. Math. J., 42(3) (1993), 10571076, MR 1254132 (94m:34157), Zbl 0819.35073.

[3] H. Crauel, A. Debussche and F. Flandoli, Random attractors, J. Dynam. Differential Equations, 9 (1997), 307-341, MR 1451294 (98c:60066), Zbl 0884.58064.

[4] H. Crauel and F. Flandoli, Attractors for random dynamical systems, Probab. Theory Related Fields, 100 (1994), 365-393, MR 1305587 (95k:58092), Zbl 0819.58023.

[5] G. Da Prato and J. Zabczyk, Stochastic Equations in Infinite Dimensions, Encyclopedia of Mathematics and its Applications, 44, University Press, Cambridge, 1992, MR 1207136 (95g:60073), Zbl 0761.60052.

[6] A. Debussche, Hausdorff dimension of a random invariant set, J. Math. Pures Appl., 77 (1998), 967-988, MR 1661029 (99k:60161) Zbl 0919.58044.

[7] A. Debussche, On the finite dimensionality of random attractors, Stochastic Anal. Appl., 15 (1997), 473-492, MR 1464401 (99i:60123), Zbl 0888.60051.

[8] J.M. Ghidaglia and R. Témam, Attractors for damped nonlinear hyperbolic equations, J. Math. Pures Appl., 66 (1987), 273-319, MR 0913856 (89m:35141), Zbl 0572.35071.

[9] A. Pazy, Semigroups of Linear Operators and Applications to Partial Differential Equations, Appl. Math. Sci., 44, Berlin, Springer-Verlag, New York, 1983, MR 0710486 (85g:47061), Zbl 0516.47023.

[10] R. Témam, Infinite-dimensional Dynamical Systems in Mechanics and Physics, Appl. Math. Sci., 68, Springer-Verlag, New York, 1988, MR 0953967 (89m:58056), Zbl 0662.35001.

Received March 24, 2003 and revised December 1, 2003. The research was supported by Key program of NNSFC under grant 10371083 and NNSFC under grant 10171072.

Department of Mathematics

SichuAN University

Chengdu, 610064

P.R. China 
Department of Applied Mathematics

University of Electronic Science and Technology

Chengdu, 610054

P.R. ChinA

E-mail address: fanxm@uestc.edu.cn 